ORIGINAL ARTICLE

\title{
Accelerated postnatal head growth follows preterm birth
}

\author{
J Cockerill, S Uthaya, C J Doré, N Modi
}

See end of article for authors' affiliations .....................

Correspondence to: Professor Modi, Faculty of Medicine, Imperial College London, Chelsea and Westminster Hospital, 369 Fulham Road, London, SW10 9NH; n.modi@ imperial.ac.uk

Accepted 29 November 2005 Published Online First 12 January 2006
Background: Poor growth after preterm birth, particularly poor head growth, is associated with impaired neurodevelopmental outcome.

Objective: To evaluate weight gain and head growth between birth and term in a contemporary cohort of preterm infants, taking into account breast milk intake and illness severity.

Methods: Subjects were inborn infants $\leqslant 32$ weeks gestation who remained in the neonatal unit up to $\geqslant 37$ weeks postmenstrual age. Weight and head circumference $(\mathrm{HC})$ were expressed as standard deviation score (SDS), growth between birth and discharge as SDS gain (SDSG), and illness severity and breast milk exposure as the number of days of level 1 (full) intensive care (\%L1IC) and the number of days on which breast milk was received (\%BM) as a percentage of days from birth to discharge.

Results: Infants showed poor postnatal weight gain but accelerated head growth. There was a highly significant fall in mean (SD) weight SDS between birth and discharge $(-0.31(0.96)$ and $-1.32(1.02)$ respectively, $\mathrm{p}<0.001)$ and a highly significant increase in HC SDS ( -0.52 (0.95) and -0.03 (1.25) respectively, $p=0.003$ ). \%LIIC had a highly significant negative impact on weight SDSG ( $p=0.006)$, and \%BM had a significant positive impact on HC SDSG ( $p=0.043$ ).

Conclusions: Accelerated postnatal head growth suggests catch up after antenatal restraint. This raises the possibility that poor neurocognitive outcomes after extremely preterm birth may in part be consequent on poor intrauterine brain growth. As postnatal head growth may be facilitated by breast milk, there is an urgent need to evaluate the optimal use of breast milk in preterm neonates. Illness severity is a significant determinant of poor postnatal weight gain.
$\mathrm{P}$ oor postnatal growth is a recognised complication of extremely preterm birth. ${ }^{1-4}$ Growth failure has mainly been reported in weight and height, with greater variation in head growth As poor growth is a recognised risk factor for adverse neurodevelopmental outcome, ${ }^{56}$ clinicians traditionally aim to promote growth and, where it has fallen behind, to accelerate growth in order to achieve catch up. This has been the stimulus for the development of nutrient enriched milk formulas and has resulted in continuing variation in the use of breast milk. However, concern has recently been raised regarding the possible adverse consequences of accelerated postnatal growth. ${ }^{7-9}$ Evidence pointing to similar associations in preterm infants ${ }^{10}$ has led to the suggestion that rapid early postnatal weight gain should be avoided..$^{11}$ The infants reported in these studies were born two to three decades ago, and nutritional regimens and the clinical profile of the preterm population have since changed substantially. We therefore aimed to evaluate the pattern of postnatal weight gain and head growth between birth and term in a contemporary cohort of preterm infants taking into account breast milk intake, birth size, illness severity, sex, and steroid exposure.

\section{METHODS}

We identified all inborn infants admitted to the neonatal unit of Queen Charlotte's \& Chelsea Hospital, London, UK, over a 30 month period from January 2002 to June 2004, who had been born at $\leqslant 32$ weeks gestation and who remained in the neonatal unit up to or later than 37 weeks postmenstrual age (term). The hospital serves an urban population and also provides a tertiary referral perinatal service. Local infants remain at Queen Charlotte's \& Chelsea until discharge, but referred infants are transferred back to their base hospital as soon as their clinical status permits.
We received approval from the local research ethics committee to extract the following information on each eligible infant from a clinical database which holds static and daily information on all admissions to the neonatal unit: sex, date of birth, gestational age at birth, birth weight, birth head circumference $(\mathrm{HC})$, date of discharge, discharge weight, discharge $\mathrm{HC}$, antenatal steroid exposure, number of days the infant received donor breast milk, number of days receiving mother's own expressed milk, number of days receiving level 1 care, and total number of days spent on the neonatal unit. Level of care assignment is based on nationally agreed criteria. ${ }^{12}$ Data required to assign level of care are captured daily and define the clinical status of the infant. Level 1 is the most intensive, and level 4 is normal care of a healthy newborn baby. Breast milk exposure (\%BM) and illness severity (\%LIIC) were defined respectively as the total number of days or part days that donor or maternal breast milk was received, and the total number of days of level 1 intensive care, each expressed as a percentage of the total number of days between birth and discharge.

We expressed weight and HC as standard deviation score (SDS) and growth between birth and age term equivalent as SDS gain (SDSG) calculated using commercially available software based on the 1990 British growth reference (Child Growth Foundation, 2 Mayfield Avenue, London W4 1PW, UK). SDSG is the change in SDS adjusted for sex and reference correlations between measurements at two time points. ${ }^{13}$ Accelerated growth is indicated by a positive SDSG, and growth along a given centile by a SDSG of 0 .

Abbreviations: $\mathrm{HC}$, head circumference; SDS, standard deviation score; SDSG, standard deviation score gain; \%LIIC, percentage level 1 intensive care; \%BM, percentage breast milk; SD, standard deviation 
Table 1 Anthropometric and hospital stay characteristics for boys and girls

\begin{tabular}{lll}
\hline & Boys ( $\mathbf{n}=\mathbf{3 7})$ & Girls $(\mathbf{n}=39)$ \\
\hline Gestational age at birth (weeks) & $28.7(2.5)$ & $28.5(1.9)$ \\
Birth weight (kg) & $1.197(0.327)$ & $1.130(0.277)$ \\
Birth weight SDS & $-0.39(0.93)$ & $-0.23(1.00)$ \\
Discharge weight (kg) & $2.710(0.525)$ & $2.709(0.450)$ \\
Discharge weight SDS & $-1.44(1.10)$ & $-1.21(0.94)$ \\
Birth head circumference (cm) & $26.5(2.0)$ & $25.9(2.0)$ \\
Birth head circumference SDS & $-0.50(0.97)$ & $-0.54(0.94)$ \\
Discharge head circumference (cm) & $34.5(1.6)$ & $34.1(1.6)$ \\
Discharge head circumference SDS & $-0.13(1.25)$ & $0.07(1.25)$ \\
Weight SDSG & $-1.48(1.31)$ & $-1.33(1.02)$ \\
Head circumference SDSG & $0.28(1.61)$ & $0.49(1.51)$ \\
Total hospital days & $73(25)$ & $74(20)$ \\
\% hospital days received breast milk & $77(22)$ & $74(26)$ \\
\% hospital days level 1 intensive care $18(19)$ & $15(13)$ \\
\hline
\end{tabular}

Values are mean (SD). There are no significant differences between boys and girls in any of the variables.

SDS, Standard deviation score; SDSG, standard deviation score gain.

\section{Nutritional intake}

The Queen Charlotte's \& Chelsea Hospital Neonatal unit has a human breast milk bank on site, and trophic feeds of fresh maternal or banked donor expressed breast milk are started within 24 hours of preterm birth. Milk volumes are increased as tolerated by a maximum of $30 \mathrm{ml} / \mathrm{kg} /$ day. If a mother chooses not to provide breast milk and refuses permission for the use of donor milk, a preterm formula (Nutriprem 1; Cow \& Gate) is used. Once a full enteral intake is established, infants receive either maternal breast milk or preterm formula. If breast milk is used, unit policy is to increase to $200 \mathrm{ml} / \mathrm{kg}$ if tolerated. Breast milk is not fortified as a matter of routine, but only if there is a decline in growth velocity despite maximum tolerated enteral feed volumes, and evidence of an anabolic state as marked by a falling blood urea. Parenteral nutrition is started within 48 hours of birth. The parenteral nutrition content of carbohydrate, protein, and lipid is increased over three days to $15 \mathrm{~g} / \mathrm{kg}$ glucose, $3 \mathrm{~g} /$ $\mathrm{kg}$ protein, and $3.5 \mathrm{~g} / \mathrm{kg}$ lipid. During the period of this study, once a combined daily enteral and parenteral intake totalling $180 \mathrm{ml} / \mathrm{kg}$ was achieved, further increments in milk volumes were accompanied by a reduction in parenteral intake of equivalent volume. Thus by the end of the first week, preterm infants were receiving about $502 \mathrm{~kJ} / \mathrm{kg} /$ day ( $120 \mathrm{kcal} / \mathrm{kg} /$ day) as a combined parenteral/enteral intake.

\section{Data analysis}

Data were analysed using SPSS version 11.5 and Stata version 8. Between group differences were evaluated using the independent samples $t$ test, and within group differences using the paired samples $t$ test. Equal variances were not assumed. Continuous variables were first examined using simple correlation and regression. Multiple regression analysis was used to examine the influence of \%BM and \%LlIC on HC SDSG and weight SDSG, allowing for HC SDS and weight SDS at birth. Evidence of an interaction between sex and breast milk exposure and sex and illness severity was sought by including an additional term in the model consisting of sex (coded 0 or 1) multiplied by either breast milk exposure or illness severity. The residuals were checked for normality.

\section{RESULTS}

We identified 489 inborn infants of $\leqslant 32$ weeks gestational age admitted from December 2001 to June 2004. There were 84 deaths before discharge in this group. Of the surviving infants, 329 were either discharged home or to their referring hospital before 37 weeks postmenstrual age, and 76 remained in our institution up to this time. Table 1 shows

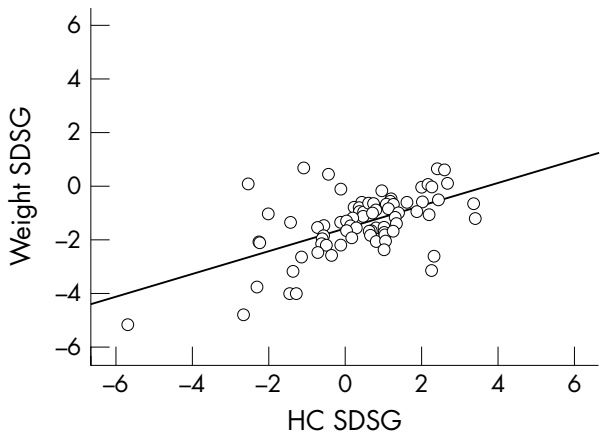

Figure 1 Growth between birth and term: weight standard deviation score gain (SDSG) in relation to head circumference (HC) SDSG (weight $\mathrm{SDSG}=-1.57+0.42 \mathrm{HC}$ SDSG; $\left.r=0.56, R^{2}=0.32, \mathrm{p}<0.001\right)$.

characteristics at birth and discharge of the 76 infants (37 boys and 39 girls). Sixty eight received antenatal steroids. No infant received postnatal steroids. There were no significant differences between boys and girls in any of the variables at birth or at discharge.

\section{Weight gain and head growth}

There was a highly significant correlation between weight SDSG and HC SDSG $(r=0.56, \mathrm{p}<0.001$; fig 1$)$. Values for HC SDSG were clustered symmetrically around zero. However, all but seven of the values for weight SDSG were below zero (range -5.16 to 0.69 ).

\section{Weight gain}

Mean weight SDSG between birth and discharge was negative (mean (SD) - 1.41 (1.17)), and the decline in weight SDS between birth and term was highly significant (mean (SD) birth weight SDS -0.31 (0.96), discharge weight SDS -1.32 (1.02); mean difference $1.01 ; 95 \%$ confidence interval (CI) 0.76 to 1.27 ; $\mathrm{p}<0.001)$. There was no significant difference in weight SDSG between infants who had and had not received antenatal steroids (mean difference $-0.22 ; 95 \% \mathrm{CI}-1.09$ to 0.66 ; $\mathrm{p}=$ 0.62 ). There was a significant inverse correlation between weight SDSG and \%LIIC ( $r=-0.32, \mathrm{p}=0.004$, two tailed $)$, but no correlation with \%BM $(r=0.09, \mathrm{p}=0.445)$. Weight SDSG was significantly predicted by illness severity $(\mathrm{B}=$ $-0.022, p=0.006$ ) allowing for birth weight SDS

\section{Head growth}

There was evidence of accelerated postnatal head growth. Mean HC SDSG between birth and discharge was positive (mean (SD) 0.39 (1.55)). There was a significant increase in HC SDS between birth and term (mean (SD) birth HC SDS -0.52 (0.95); discharge HC SDS -0.03 (1.25); mean difference $-0.49 ; 95 \% \mathrm{CI}-0.81$ to $-0.18 ; \mathrm{p}=0.003)$, and there was a significant inverse relation between birth HC SDS and HC SDSG $(r=-0.23, \mathrm{p}=0.049)$. There was no significant difference in HC SDSG between infants who had and had not received antenatal steroids (mean difference $-0.18 ; 95 \%$ CI -1.48 to $1.11 ; \mathrm{p}=0.76)$. There was no evidence of any impact of illness severity on HC SDSG ( $r=$ $-0.12, \mathrm{p}=0.32$ ). Multiple regression analysis showed a significant positive impact of breast milk $(\mathrm{B}=0.015, \mathrm{p}=$ 0.043 ) on HC SDSG, allowing for birth HC SDS (B $=-0.443$, $\mathrm{p}=0.019)$. We found no significant differences between the sexes when these analyses were repeated including sex in the regression model, nor any evidence of an interaction between sex and breast milk exposure $(p=0.76)$, or sex and illness severity $(p=0.58)$. We also repeated these analyses excluding data from an infant with trisomy 21 in whom postnatal head growth had been extremely poor (HC SDSG -5.7), but our conclusions were unaltered. 


\section{DISCUSSION}

We have made the novel observation in this contemporary cohort of preterm infants that, although weight gain was poor, there was evidence of accelerated postnatal head growth. This was indicated by the significant increase in HC SDS between birth and term equivalent and the inverse relation between birth HC SDS and HC SDSG. This observation is in keeping with our previously reported finding that, by term, infants born below 32 weeks gestation have no significant difference in HC SDS when compared with term born infants. ${ }^{14}$ As accelerated growth occurs in response to escape from restraint, the implication is that infants born preterm have experienced restraint in head growth in utero. This raises the possibility that poor neurocognitive outcomes after extremely preterm birth may at least in part be consequent on poor intrauterine brain growth.

Accelerated postnatal head growth was enhanced by exposure to breast milk. This contrasts with the slower weight gain of breast fed infants than formula fed infants..$^{15} 16$ Despite this, breast fed infants have better cognitive outcomes, ${ }^{17}$ an effect that persists after adjustment for socioeconomic variables. The benefits of breast feeding on cognitive outcomes appear to be even greater in low birthweight infants, ${ }^{17}$ although the magnitude of this effect is not known with certainty. We were unable to quantify the precise volumes of breast milk received. However, as the composition of breast milk is very variable, in the absence of detailed composition analyses, the volume of breast milk is an imprecise indication of actual nutritional intake. We also acknowledge that the $95 \%$ CI for the regression coefficient of \%BM on HC SDSG indicated that, for every per cent increase in breast milk exposure, the true impact on HC SDSG may lie anywhere between 0 and 0.029 and indicates the need to confirm our observations.

The basis for the benefits of breast milk on brain growth and development remain a matter of conjecture, although the greater content of long chain polyunsaturated fatty acids is a prime candidate. ${ }^{17}$ The human brain is $60 \%$ lipid, and the long chain polyunsaturated fatty acids docosahexanoic acid and arachidonic acid are major constituents. ${ }^{18}$ Preterm infants are deprived of the third trimester transplacental supply and are particularly vulnerable to a deficit of long chain polyunsaturated fatty acids. ${ }^{19}$ Although breast milk is advocated for all newborns, a number of factors contribute to very variable and often low rates of breast milk use for preterm infants. ${ }^{20}$ For any individual mother, the difficulties inherent in initiating and sustaining lactation after the birth of an extremely preterm infant are well known. Even when a mother chooses to provide breast milk, she is on average able to supply only about half of her infant's needs. ${ }^{21}$ The emergence of HIV/AIDS, the practical difficulties in screening donors for transmissible infections, and the costs of pasteurising and storing milk have led to a decline in human milk banking and the availability of donor breast milk. The use of fresh maternal breast milk poses other infective risks such as the transmission of cytomegalovirus, which may lead to serious illness in the preterm neonate. ${ }^{22}$

Concerns also persist that breast milk may be inadequate to meet the nutritional demands of the preterm infant. In this respect the results of clinical trials initiated two decades ago demonstrating greater growth with formula milk compared with unfortified term human milk ${ }^{23}$ have been highly influential. Clinicians traditionally aim to promote growth and, where it has fallen behind, to accelerate growth in order to achieve catch up. In neonatal medicine, the close association between poor growth and adverse neurodevelopmental outcome has given added impetus to this practice.

Centile charts based on cross sectional data from newborn infants are widely used as a template against which to monitor postnatal growth even though it is recognised that infants born preterm weigh less than intrauterine fetuses at the same gestational age. ${ }^{24}$ As fetal weights determined ultrasonographically and newborn weights converge to the same value at term, growth rates based on cross sectional newborn data are more rapid than fetal growth rates and in effect are projected to achieve catch up growth by term. Nutritional support that aims to achieve the trajectory of cross sectional growth charts derived from newborn measurements will thus also aim to promote catch up growth, and it has been suggested that this should be the basis on which recommended dietary intakes should be calculated. ${ }^{25}$ Breast milk, even in maximum tolerated volumes, would not meet these requirements. This, together with the difficulties in obtaining breast milk, has led many clinicians to rely on nutrient enriched milk formula or breast milk fortification for preterm infants.

Although superior growth has been widely considered desirable and growth performance is used to assess nutritional outcomes ${ }^{26}{ }^{27}$ there is increasing concern that catch up or accelerated growth in infancy may have adverse long term consequences, particularly in relation to obesity, insulin resistance, cardiovascular disease, and dyslipidaemia. ${ }^{7-9}$ In a series of papers examining outcomes of preterm infants recruited to randomised trials in the 1980s, Singhal et al have shown that greater intake of breast milk is associated with slower neonatal growth rates but improved indices of cardiovascular health, ${ }^{10} 28$ insulin resistance, ${ }^{11}$ and lipid profile in adolescence. ${ }^{29}$ Preterm infants are very likely to have suffered both antenatal and postnatal growth restraint, and the active promotion of catch up growth may further increase their vulnerability. ${ }^{29-31}$ This has led to the suggestion that postnatal nutrient restriction may be beneficial. ${ }^{11}$

We found no evidence of accelerated postnatal weight gain between birth and term despite the early introduction of combined enteral/parenteral nutrition. On the contrary, postnatal weight gain was poor. It did not appear to be influenced by breast milk, but illness severity had a highly significant adverse impact. Although there was a significant positive association between HC SDSG and weight SDSG, in contrast with HC SDSG, all but seven of the infants had a weight SDSG below zero. This is in keeping with the notion of head sparing in the face of adverse influences on growth.

We have previously shown that, by term, infants born below 32 weeks gestation, although smaller than term born counterparts, have an identical fat mass/fat-free mass ratio, suggesting that poor postnatal growth is not nutritionally

\section{What is already known on this topic}

- Although recommended for all newborn infants, the slower growth of preterm infants fed breast milk has contributed to the wide variation in its use

- The optimal rate of postnatal growth after preterm birth is unknown

\section{What this study adds}

- Despite slow somatic growth, infants born extremely preterm show accelerated postnatal head growth that is enhanced by breast milk

- This raises the possibility that breast milk may have a modulatory impact on brain growth and development 
determined. $^{14}$ Others have similarly suggested that nonnutritional factors are important and account for around half of the variance in postnatal weight gain. ${ }^{32}$ Possible nonnutritional determinants include increased energy expenditure, $^{33}$ glucocorticoid excess, increased catecholamines, and chronic inflammation. ${ }^{34}$ Our present study indicates that it must be recognised that poor postnatal weight gain may also be a consequence of illness severity.

Paradoxically, the poor postnatal weight gain of preterm infants may be protective against adverse long term metabolic consequences. Future improvements in perinatal care and reduced illness severity may reverse this situation and result in accelerated postnatal weight gain. This argues for the need for long term metabolic follow up of infants born preterm.

We suggest that evaluation of the optimal use of human breast milk for preterm neonates is urgently needed. This should address the need for fortification, the treatment and storage of milk, cost-benefit analyses of maternal lactation support and breast milk banking, and the assessment of cognitive and metabolic outcomes in addition to growth.

\section{ACKNOWLEDGEMENTS}

We gratefully acknowledge the invaluable assistance of Ian de Vega in data extraction.

\section{Authors' affiliations}

J Cockerill, S Uthaya, N Modi, Imperial College London, Chelsea \& Westminster Campus, 369 Fulham, Road, London SW10 9NH, UK S Uthaya, N Modi, Queen Charlotte's \& Chelsea Hospital, Du Cane Road, London W12 ONN, UK

C J Doré, Medical Research Council Clinical Trials Unit, 222 Euston Road, London NWI 2DA, UK

Competing interests: none declared

\section{REFERENCES}

1 Finnstrom O, Otterblad Olausson P, Sedin G, et al. Neurosensory outcome and growth at three years in extremely low birthweight infants: follow-up results from the Swedish national prospective study. Acta Paediatr 1998;87:1055-60.

2 Bucher HU, Killer C, Ochsner Y, et al. Growth, developmental milestones and health problems in the first 2 years in very preterm infants compared with term infants: a population based study. Eur J Pediatr 2002;161:151-6.

3 Clark RH, Thomas P, Peabody J. Extrauterine growth restriction remains a serious problemin prematurely born neonates. Pediatrics 2003;11 1:986-90.

4 Wood NS, Costeloe K, Gibson AT, et al. The EPICure study: growth and associated problems in children born at 25 weeks of gestational age or less. Arch Dis Child Fetal Neonatal Ed 2003;88:F492-500.

5 Hack M, Breslau N. Very low birth weight infants: effects of brain growth during infancy on intelligence quotient at 3 years of age. Pediatrics 1986:77:196-202.

6 Latal-Hajnal B, von Siebenthal K, Kovari H, et al. Postnatal growth in VLBW infants: significant association with neurodevelopmental outcome. J Pediatr 2003; 143: 163-70.

7 Ong KK, Ahmed ML, Emmett PM, et al. 2000 Association between postnatal catch-up growth and obesity in childhood: prospective cohort study. BMJ 2000;320:967-71.
8 Soto N, Bazaes RA, Pena V, et al. Insulin sensitivity and secretion are related to catch-up growth in small-for-gestationalage infants at age 1 year: results from a prospective cohort. J Clin Endocrinol Metab 2003;88:3645-50.

9 Hales CN, Ozanne SE. The dangerous road of catch-up growth. J Physiol 2003;547:5-10

10 Singhal A, Cole TJ, Fewtrell $M$, et al. Is slower early growth beneficial for longterm cardiovascular health? Circulation 2004;109:1108-13.

11 Singhal A, Fewtrell M, Cole TJ, et al. Low nutrient intake and early growth for later insulin resistance in adolescents born preterm. Lancet 2003;361:1089-97.

12 British Association of Perinatal Medicine. Standards for hospitals providing neonatal intensive care and high dependency care, and categories of babies requiring neonatal care, 2 nd ed. London: British Association of Perinatal Medicine, 2001

13 Cole TJ, Freeman JV, Preece MA. British 1990 growth reference centiles for weight, height, body mass index and head circumference fitted by maximum penalized likelihood. Stat Med 1998;17:407-29.

14 Uthaya S, Thomas EL, Hamilton G, et al. Altered adiposity after extremely preterm birth. Pediatr Res 2005;57:211-15.

15 Ong KK, Preece MA, Emmett PM, et al. Size at birth and early childhood growth in relation to maternal smoking, parity and infant breastfeeding: longitudinal birth cohort study and analysis. Pediatr Res 2002;52:863-7.

16 Kramer MS, Guo T, Platt RW, et al. Feeding effects on growth during infancy. $J$ Pediatr 2004;145:600-5.

17 Anderson JW, Johnstone BM, Remley DT. Breast-feeding and cognitive development: a meta-analysis. Am J Clin Nutr 1999;70:525-35.

18 Crawford MA The role of essential fatty acids in neural development: implications for perinatal nutrition. Am J Clin Nutr 1993;57(suppl):703S-10S.

19 Fleith M, Clandinin MT. Dietary PUFA for preterm and term infants: review of clinical studies. Crit Rev Food Sci Nutr 2005;45:205-29.

20 Espy KA, Senn TE. Incidence and correlates of breast milk feeding in hospitalized preterm infants. Soc Sci Med 2003;57:1421-8.

21 Lucas A, Fewtrell MS, Morley R, et al. Randomized outcome trial of human milk fortification and developmental outcome in preterm infants. Am J Clin Nutr 1996;64:142-51.

22 Maschmann J, Hamprecht K, Dietz K, Cytomegalovirus infection of extremely low-birth weight infants via breast milk, et al. Clin Infect Dis 2001;33:1998-2003

23 Henderson G, Anthony MY, McGuire W. Formula milk versus term human milk for feeding preterm or low birth weight infants. Cochrane Database of Systematic Reviews. Issue 4. 2001.

24 Lackman F, Capewell V, Richardson B, et al. The risks of spontaneous preterm delivery and perinatal mortality in relation to size at birth according to fetal versus neonatal growth standards. Am J Obstet Gynaecol 2001;184:946-53.

25 Cooke RJ. Nutrient requirements in preterm infants. Pediatr Res 2003;53:2.

26 Bloom BT, Mulligan J, Arnold C, et al. Improving growth of very low birth weight infants in the first 28 days. Pediatrics 2003;112:8-14.

27 Cooke RJ, Ainsworth SB, Fenton AC. Postnatal growth retardation: a universal problem in preterm infants. Arch Dis Child Fetal Neonatal Ed 2004;89:F428-30.

28 Singhal A, Cole TJ, Lucas A. Early nutrition in preterm infants and later blood pressure: two cohorts after randomised trials. Lancet 2001;357:413-19.

29 Singhal A, Cole TJ, Fewtrell M, et al. Breastmilk feeding and lipoprotein profile in adolescents born preterm: follow-up of a prospective randomised study. Lancet 2004;363:1571-8.

30 Mortaz M, Fewtrell MS, Cole TJ, et al. Birth weight, subsequent growth, and cholesterol metabolism in children 8-12 years old, born preterm. Arch Dis Child 2001;84:212-17.

31 Hofman PL, Regan F, Jackson WE, et al. Premature birth and later insulin resistance. N Eng J Med 2004;351:2179-86.

32 Embleton NE, Pang N, Cooke RJ. Postnatal malnutrition and growth retardation: an inevitable consequence of current recommendations in preterm infants? Pediatrics 2001;107:270-3.

33 Baver J, Hentschel R, Linderkamp O. Effect of sepsis syndrome on neonatal oxygen consumption and energy expenditure. Pediatrics 2002;1 10:e69.

34 Roth SM, Schrager MA, Lee MR, et al. Interleukin-6 genotype is associated with fat-free mass in men but not women. J Gerontol A Biol Sci Med Sci 2003;58:B1085-8. 